\title{
UM MODELO APROXIMADO COM REAÇÃO DA DISPERSÃO DE GASES DE UMA CENTRAL TERMOELÉTRICA
}

\author{
E. A. S. CHIARAMONTE \\ ${ }^{1}$ Universidade Federal do Pampa, Campus Bagé, Engenharia Química \\ E-mail para contato: edsonchi@ portoweb.com.br
}

\begin{abstract}
RESUMO - O problema da poluição atmosférica, a propagação dos gases emitidos e os impactos ao meio ambiente têm chamado a atenção. A propagação de gases emitidos de uma central termoelétrica, com a queima de carvão, é apresentada neste trabalho. As vazões da chaminé são descritas pelo "AP-42" - Compilação dos Fatores de Emissão de Poluentes Atmosféricos do "Environmental Protection Agency (EPA)" dos Estados Unidos. Um programa de computador está sendo desenvolvido e foi usado para descrever a fonte de emissão e a propagação dos gases. O modelo aproximado separa o mecanismo de propagação (advecção e difusão) e o de reação química. Um mecanismo químico simplificado separando a química dos gases de Nitrogênio $\left(\mathrm{NO}_{\mathrm{x}}\right)$ e a dos compostos orgânicos voláteis ("VOC") é discutida. Os resultados numéricos são apresentados para as concentrações dos gases emitidos dessa chaminé.
\end{abstract}

\section{INTRODUÇÃO}

Nas últimas décadas, a poluição atmosférica tem chamado atenção devido ao seu efeito nocivo para o ambiente. $\mathrm{O}$ estudo da dispersão e do transporte de gases na atmosfera tem chamado a atenção de muitos grupos de pesquisa. Uma das fontes de emissões de gases são as chaminés industriais, Fenger (1999). Estes gases podem sofrer transformações químicas na atmosfera e produzirem produtos que irão interagir com o ambiente. Por exemplo, os Óxidos de Enxofre $\left(\mathrm{SO}_{\mathrm{x}}\right)$ e de Nitrogênio $\left(\mathrm{NO}_{\mathrm{x}}\right)$ são precursores de chuva ácida e o Dióxido de Nitrogênio $\left(\mathrm{NO}_{2}\right)$ produz o gás Ozônio presente na baixa atmosfera, Seinfeld e Pandis (2006). A propagação dos gases emitidos, de uma central termoelétrica de carvão, é representada por um modelo aproximado. Nesse trabalho é descrito o modelo de propagação, dispersão e reação química. Também são mostrados os resultados numéricos obtidos para as concentrações dos gases Ácido Nitrico $\left(\mathrm{HNO}_{3}\right)$, Dióxido de Nitrogênio $\left(\mathrm{NO}_{2}\right)$, Óxido Nitroso (NO) e Ozônio $\left(\mathrm{O}_{3}\right)$ gerados a partir da fonte de emissão, na direção do vento.

\section{O MODELO APROXIMADO DE PROPAGAÇÃO E REAÇÃO QUÍMICA}

\subsection{A Fonte de Emissão da Central Termoelétrica de Carvão}

Os principais gases envolvidos com a poluição atmosférica NOx, SOx, monóxido de Carbono (CO) e os Compostos Orgânicos Voláteis (COV) têm suas vazões estimadas a partir dos fatores de 
emissão do inventário “AP-42”, USEPA (2012). As vazões, dos gases que saem na chaminé, são estimadas pela relação:

$$
Q_{i, E}=A_{i} \times E_{F, i} \times\left(1-\frac{E_{R, i}}{100}\right)
$$

Onde $Q_{i, E}$ é a vazão do composto " i” $\left(\mathrm{kg} \mathrm{h}^{-1}\right)$ obtido pelos fatores de emissão, $\mathrm{A}_{\mathrm{i}}$ é a taxa de consumo do combustível $\left(\mathrm{kg} \mathrm{h}^{-1}\right), \mathrm{E}_{\mathrm{F}, \mathrm{i}}$ é o fator de emissão do gás “i” ( $\mathrm{kg}$ do gás “i”/kg total) e $\mathrm{E}_{\mathrm{R}, \mathrm{i}}$ é o fator de eliminação do gás, no equipamento de remoção da poluição. As vazões dos demais gases são determinadas pelas relações estequiométricas, o valor do excesso de ar empregado e da análise elementar do Carvão de Candiota, mostrada na Tabela 1, conforme Rodrigues (2004). A quantidade de cada gás gerado por kg de carvão, a partir de cada elemento presente no carvão, por exemplo, o Dióxido de Carbono $\left(\mathrm{CO}_{2}\right)$ é obtido a partir do elemento Carbono (C), é obtida com a Equação a seguir

$$
\left(X_{g i}\right)_{C}=\frac{M_{g i}}{M_{i}}\left(X_{i}\right)_{u m}
$$

Onde $\left(X_{g i}\right)_{C}$ é a quantidade do gás " $g i$ " em kg por kg de carvão, $M_{g i}$ é a massa molar do gás que sai na chaminé e $M_{i}$ é a massa molar do elemento "i" que reage com o oxigênio. A vazão, dos demais gases, são dados pela relação

$$
Q_{i, D}=\left(X_{g i}\right)_{C} A_{i}
$$

Onde $Q_{i, D}$ é a vazão dos demais gases.

Tabela 1. Dados elementares do carvão.

\begin{tabular}{|l|c|}
\hline \multicolumn{1}{|c|}{ Compostos } & \% mássica \\
\hline $\mathrm{H}_{2} \mathrm{O}$ & 3,58 \\
\hline Material Volátil & 19,77 \\
\hline Carbono fixo & 25,66 \\
\hline Cinzas & 54,57 \\
\hline Enxofre & 2,04 \\
\hline Carbono & 32,26 \\
\hline Hidrogênio & 2,13 \\
\hline Nitrogênio & 0,67 \\
\hline Oxigênio + halogênios & 8,33 \\
\hline
\end{tabular}




\subsection{O Modelo de Propagação com Reação Química}

O modelo de propagação (advecção, dispersão e reação química) dos gases emitidos da chaminé adota uma liberação instantânea (denominada "puff"), de formato retangular, que separa os mecanismos de reação química e de propagação a cada tempo de deslocamento do "puff", Orlandi (2004). 0 modelo de reação química adota a variação da concentração dos gases da mistura, num volume constante do "puff", a cada tempo de deslocamento. E, o modelo de propagação é um modelo aproximado que aplica a diluição da massa total de gases liberada no final do tempo de deslocamento. 0 modelo de diluição usa uma solução da equação da difusão para a propagação da massa total de gases para avaliar a entrada de ar a cada tempo de deslocamento. Esses modelos são apresentados a seguir.

\section{Modelo de reações químicas simplificadas para a obtenção do $\mathrm{HNO}_{3}$}

Nesse trabalho foi adotado um modelo simplificado para a avaliação do Ácido Nítrico formado na atmosfera a partir dos compostos de Nitrogênio liberados numa Central Termoelétrica. 0 conjunto de reações foi desacoplado das reações dos Compostos Orgânicos Voláteis (VOC) para uma avaliação dos resultados obtidos e uma maior facilidade de solução do conjunto de equações diferenciais ordinárias que apresentam problema de instabilidade numérica, conforme Yamaryino et al. (1992). 0 conjunto de reações usado é mostrado:
1) $\mathrm{NO}_{2}+h v \stackrel{\mathrm{O}_{2}}{\rightarrow} \mathrm{NO}+\mathrm{O}_{3}$
$R_{1}=J_{1} C_{N O_{2}}$
2) $\mathrm{O}_{3}+h v \rightarrow \mathrm{O}_{2}+\mathrm{O}^{\prime}$

$$
R_{2}=J_{2} C_{O_{\mathrm{s}}}
$$
3) $\mathrm{O}_{3}+\mathrm{NO} \rightarrow \mathrm{NO}_{2}+\mathrm{O}_{2}$
$R_{3}=k_{3} C_{O_{3}} C_{N o}$
4) $\mathrm{H}_{2} \mathrm{O}+\mathrm{O}^{\prime} \rightarrow 2 \mathrm{OH}$
$R_{4}=k_{4} C_{H_{2}} O C_{O r}$
5) $\mathrm{OH}+\mathrm{NO}_{2} \rightarrow \mathrm{HNO}_{3}$

$$
R_{5}=k_{5} C_{O H} C_{N O_{2}}
$$

Esse conjunto de reações forma o seguinte conjunto de equações diferenciais ordinárias, que descrevem o balanço de massa num sistema de volume constante, para os gases,

$$
\begin{aligned}
& \frac{d C_{\mathrm{NO}_{\mathrm{n}}}}{d t}=-J_{1} C_{\mathrm{NO}_{2}}+k_{3} C_{\mathrm{NO}} C_{\mathrm{O}_{\mathrm{g}}}-k_{5} C_{\mathrm{NO}_{2}} C_{\mathrm{OH}} \\
& \frac{d C_{\mathrm{NO}}}{d t}=J_{1} C_{\mathrm{NO}_{2}}-k_{3} C_{\mathrm{NO}} C_{\mathrm{O}_{\mathrm{g}}}
\end{aligned}
$$




$$
\begin{aligned}
& \frac{d C_{O_{3}}}{d t}=J_{1} C_{\mathrm{NO}_{2}}-J_{2} C_{\mathrm{O}_{\mathrm{s}}}-k_{3} C_{\mathrm{NO}} C_{\mathrm{O}_{\mathrm{s}}} \\
& \frac{d C_{\mathrm{O}^{\prime}}}{d t}=J_{2} C_{\mathrm{O}_{\mathrm{a}}}-k_{4} C_{\mathrm{O}^{\prime}} C_{\mathrm{H}_{2} \mathrm{O}} \\
& \frac{d C_{\mathrm{H}_{2} \mathrm{O}}}{d t}=-k_{4} C_{\mathrm{O}^{\prime}} C_{\mathrm{H}_{2} \mathrm{O}} \\
& \frac{d C_{\mathrm{OH}}}{d t}=2 k_{4} C_{\mathrm{O}^{\prime}} C_{\mathrm{H}_{2} \mathrm{O}}-k_{5} C_{\mathrm{NO}_{2}} C_{\mathrm{OH}} \\
& \frac{d C_{\mathrm{HNO}}}{d t}=k_{5} C_{\mathrm{NO}_{2}} C_{\mathrm{OH}} \\
& \frac{d C_{\mathrm{O}_{3}}}{d t}=-J_{1} C_{\mathrm{NO}_{2}}+J_{2} C_{\mathrm{O}_{\mathrm{s}}}+k_{3} C_{\mathrm{O}_{2}} C_{\mathrm{NO}}
\end{aligned}
$$

\section{Modelo de Reações Químicas}

O sistema das sete equações ordinárias acopladas, para os sete componentes, descreve o balanço de massa dos componentes da química do $\mathrm{HNO}_{3}$ num volume constante, isto é tratado independente da dispersão atmosférica (diluição com o ar atmosférico). 0 sistema é resolvido a cada tempo de deslocamento do "puff" pelo método implícito de integração numérica "Backward Differentiation Formula" BDF de quarta ordem

$$
y_{n}=\frac{48}{25} y_{n-1}-\frac{36}{25} y_{n-2}+\frac{16}{25} y_{n-3}-\frac{3}{25} y_{n-4}+h \frac{12}{25} f\left(y_{n}, t_{n}\right)
$$

Onde $y_{n}$ é o valor da função no tempo " $t_{n}$ ", $h$ é o passo de integração numérico e $f\left(y_{n}, t_{n}\right)$ é a função derivada de $y$ calculada no tempo atual. 0 passo de integração é um valor muito pequeno devido aos problemas de rigidez, pela diferença das velocidades de reações dos componentes, reações mais rápidas e reações lentas. A discretização gera um sistema de equações não lineares para as concentrações. Ele é resolvido pelo método de substituição sucessiva a cada passo de integração.

\section{$\underline{\text { O modelo de advecção e dispersão do "Puff" }}$}

A liberação instantânea é aproximada por um modelo de propriedades homogêneas, denominados modelos de caixa, conforme Seinfeld e Pandis (2006). É adotada a forma de prisma com base retangular para esse "puff", conforme a Figura 1. Uma solução local da equação da difusão é usada para determinar o perfil de concentração e a entrada de ar para o "puff". 


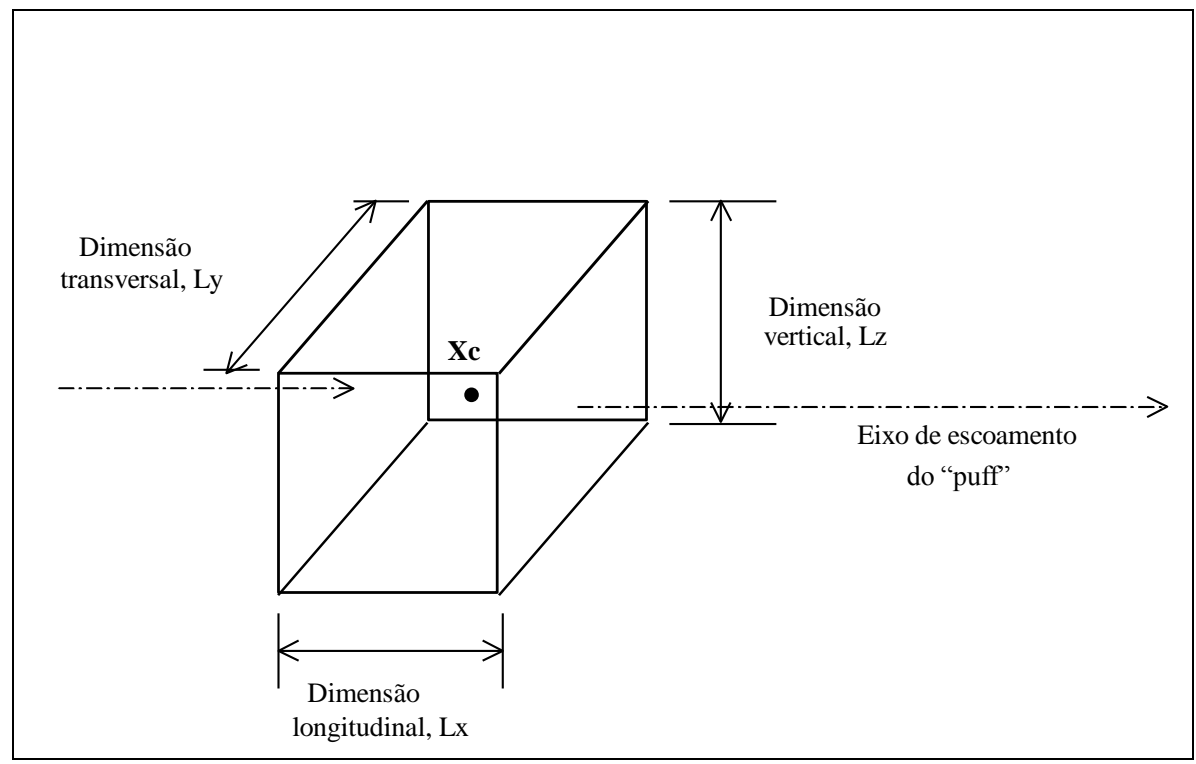

Figura 1 -"Puff" de prisma com base retangular.

O "puff" possui as seguintes variáveis que o caracterizam: a massa total de gás $\left(m_{g p}\right)$, a posição do seu centro em relação à fonte de emissão $\left(X_{C, i}\right)$, a dimensão longitudinal $\left(L_{x, i}\right)$, a dimensão transversal $\left(L_{y, i}\right)$, a dimensão vertical $\left(L_{z, i}\right)$, o tempo de deslocamento na atmosfera $\left(t_{p}\right)$, e o tempo total de liberação desde a fonte $\left(t_{t}\right)$.

O volume do "puff" de gás é determinado por:

$$
V_{i}=L_{x, i} L_{y, i} L_{z, i}
$$

Onde i refere-se ao tempo de deslocamento "i".

A concentração total de todos os gases, no "puff", é dada pela relação:

$$
\rho_{g p, i}=\frac{m_{g p, i}}{L_{x, i} L_{y, i} L_{z i, i}}
$$

Onde $m_{g p, i}$ é a massa total de gases.

A posição do centro "puff" é determinada a cada deslocamento de tempo do "puff" por: 


$$
x_{C_{r} i}=x_{C_{r} i-1}+u_{m, i} t_{p}
$$

Onde $\mathrm{x}_{\mathrm{C}, \mathrm{i}}$ é a posição do centro do "puff", $u_{m, i}$ a velocidade média de deslocamento do "puff" (obtida a partir do perfil médio logarítmico de velocidade) e $t_{p}$ o intervalo de tempo de deslocamento do "puff".

O tempo de deslocamento do "puff" foi adotado igual ao tempo de liberação na fonte de emissão, que é o quociente da massa de gás liberada pela vazão

$$
t_{p}=\frac{m_{g p, 0}}{Q}
$$

O "puff" de gás incorpora uma quantidade de ar, a cada intervalo de deslocamento, e tem as suas dimensões corrigidas pelas relações:

$$
\begin{aligned}
& L_{x, i}=L_{x_{i} i-1}+\Delta x_{i} \\
& L_{y, i}=L_{y, i-1}+\Delta y_{i} \\
& L_{z, i}=L_{z, i}-1+\Delta z_{i}
\end{aligned}
$$

Onde $\Delta x, \Delta y$ e $\Delta z$ são os aumentos das dimensões longitudinal, transversal e vertical, respectivamente.

\section{O modelo de entrada de ar para o "puff"}

A variação da concentração dos componentes no "puff" devido à difusão dos componentes na atmosfera é avaliada com um novo procedimento a partir da entrada de ar atmosférico para o seu interior. A concentração média total da massa de gás do "puff", com o tempo de deslocamento, é dada por:

$$
\rho_{\mathrm{gp}, \mathrm{m}}=\frac{\mathrm{m}_{\mathrm{gp}}}{\mathrm{v}_{1-1}+\Delta \mathrm{v}_{1}}
$$

Onde $\rho_{g p, m}$ é a concentração total média do "puff", $V_{i}$ é o volume anterior do "puff" e $\Delta V_{i}$ é o volume de ar que entrou no "puff" no tempo de deslocamento. Então, o volume de entrada de ar é determinado a partir da concentração média

$$
\Delta V_{\mathrm{i}}=\frac{m_{g p}-\rho_{g p, m} V_{i-1}}{\rho_{g p, m}}
$$


Esse procedimento faz a conexão do modelo aproximado de "puff" retangular com uma solução particular da equação da difusão para a emissão total.

\section{METODOLOGIA}

A metodologia empregada foi aplicar o modelo descrito. Ele foi implementado num programa de computador, em linguagem Fortran, com o software livre Force plus Fortran 90. O programa faz as leituras dos dados de caracterização do carvão, da fonte de emissão, da composição da atmosfera e das características meteorológicas do vento e executa o modelo de propagação com reação química.

\section{RESULTADOS OBTIDOS COM O MODELO}

O modelo foi empregado para uma fonte de emissão, conforme os dados citados pela empresa CGTEE (2012) para a denominada fase "B", para um dia ensolarado e com as características de atmosfera instável. Esses dados são mostrados na Tabela 2. Os resultados são mostrados nos gráficos da Figuras 2. Os gráficos mostram os valores de concentração, no eixo das ordenadas, contra a distância do ponto de emissão, no eixo da abscissa. A Figura também mostra o volume de entrada de ar por diluição.

Tabela 2. Dados usados na simulação.

\begin{tabular}{|l|c|}
\hline \multicolumn{2}{|c|}{ Dados da Fonte de emissão } \\
\hline Vazão, em kg s$^{-1}$ & 0,275560 \\
\hline Altura da chaminé, em m & 150,0 \\
\hline Diâmetro da chaminé, em m & 5,0 \\
\hline \multicolumn{2}{|c|}{ Dados da atmosfera } \\
\hline Pressão, em atm & 1,0 \\
\hline Temperatura, em ${ }^{\circ} \mathrm{C}$ & 25,0 \\
\hline Umidade relativa do ar, em \% & 30,0 \\
\hline Velocidade do vento a 10 m, em m s${ }^{-1}$ & 7,0 \\
\hline Velocidade convectiva, $\mathrm{m} \mathrm{s}^{-1}$ & 1,64 \\
\hline $\begin{array}{l}\text { Comprimento da camada limite convect } \\
\text { em m }\end{array}$ & 780,0 \\
\hline
\end{tabular}



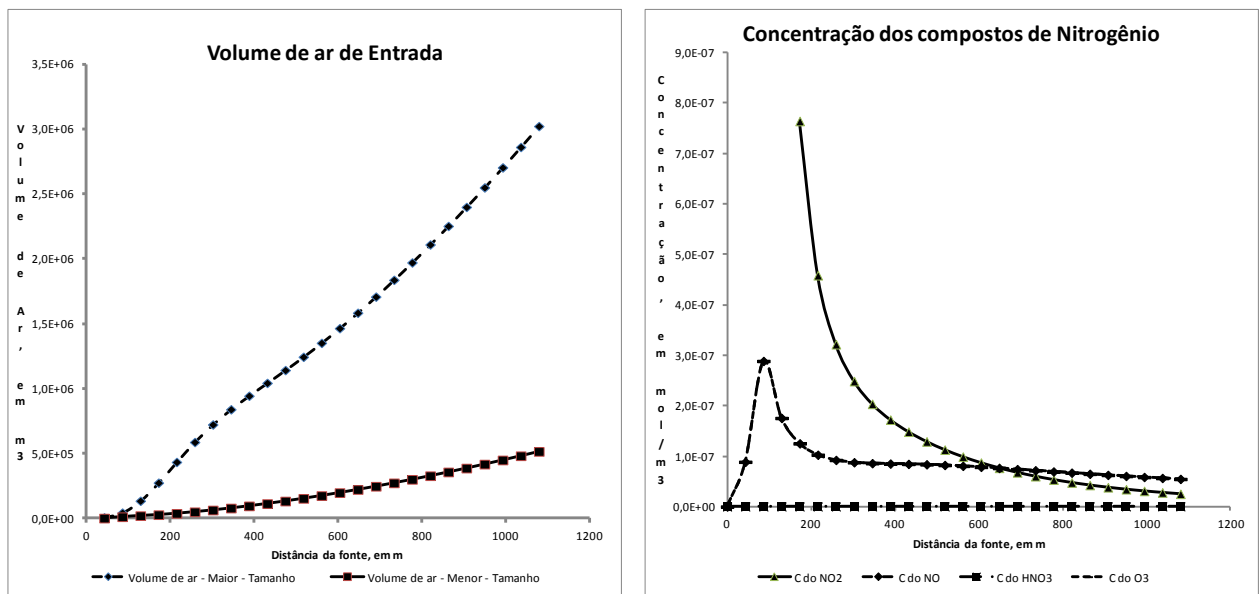

Figura 3. Volume de entrada de ar por difusão e perfil de concentração dos compostos de Nitrogênio.

\section{CONCLUSÕES}

Esse modelo aproximado que separa os mecanismos de dispersão global e do de reação química mostra-se adequado, devido a solução tornar-se mais rápida. O modelo continuará a ser desenvolvido e mais aplicações serão realizadas.

\section{REFERÊNCIAS}

FENGER, J. Urban Air Quality. Atmospheric Environment. 33:12, p. 4877-4900, 1999.

SEINFELD, J. \& PANDIS, S. Atmospheric Chemistry and Physics: From Air Pollution to Climate Change. John Wiley \& Sons, New Jersey, 2006.

AP-42. Compilação dos Fatores de Emissão de Poluentes Atmosféricos. Disponível em:<http://www.epa.gov/ttn/chief/ap42/>. Acesso em 13 de setembro de 2011 ás 10:20.

RODRIGUES, C. P. Modelagem e Simulação da Câmara de Reação de uma Caldeira a Carvão Pulverizado. Dissertação de Mestrado. Programa de Pós-Graduação em Engenharia Química, UFRGS, 110 p., 2004.

ORLANDI, L. Um Modelo Aproximado de "Puffs" para Representar a Dispersão de Espécies na Atmosfera num Ambiente Industrial. Dissertação de Mestrado. Programa de Pós- Graduação em Engenharia Energia, Ambiente e Materiais. Ulbra, 90 p., 2004.

YAMARTINO, R. J., SCIRE, J. S., CARMICHAEL, G. R., CHANG, Y. S. The CALGRID Mesoscale Photochemical Grid Model I, Model Formulation. Atmospheric Environment, 26A, p. 1493-1512, 1992.

CGTEE. Companhia de geração Térmica de energia Elétrica. Disponível em <http://www.cgtee.gov.br/sitenovo/index.php>. Acesso em 13 de setembro de 2012 ás 10:05. 